\title{
Cartesian trajectory tracking for manipulators using optimal control theory $\dagger$
}

\author{
OLAV EGELAND $\ddagger$
}

Keywords: Robots; kinematically redundant manipulators; optimal control; tracking systems.

\begin{abstract}
A Cartesian trajectory tracking system for manipulators is developed using optimal control theory. By including the Cartesian position in the state vector, transformation of the trajectory from Cartesian space to manipulator joint space is avoided, and the Jacobian matrix need not be inverted. The tracking system may also be applied to kinematically redundant manipulators. For this type of manipulator, singularities are avoided by choosing a suitable performance index in the optimal control problem. Simulation using a simple kinematically redundant manipulator shows that a small tracking error can be achieved with low motor torques.
\end{abstract}

\section{Introduction}

In certain applications such as seam welding, the robot hand is required to track a trajectory specified in terms of velocity and position referred to the Cartesian non-moving base system. In other applications, feedback from vision systems or proximity sensors may be used to obtain a desired position and rotation of the hand relative to an object in the environment. Here the position of the hand is conveniently expressed in Cartesian coordinates, while the rotation may be given in terms of Euler angles.

The conventional solution to the problem of tracking a trajectory specified in Cartesian coordinates is to transform the trajectory to joint coordinates and then track this reference (Luh 1983, Saridis 1983, Paul 1979, Taylor 1979, Whitney 1972).

The transformation from Cartesian to joint coordinates is relatively complex, but as a rule it may be carried out for commercially available robots with 5 or 6 degrees of freedom. For kinematically redundant manipulators, a transformation from Cartesian to joint coordinates can only be carried out by specifying a suitable criterion which is to be minimized.

Tarn, Bejczy, Isidori and Chen (1984) and Luh, Walker and Paul (1980a) formulate the tracking problem in the task space, and obtain a linear time invariant state space model by means of exact compensation of system nonlinearities. However, these techniques require the inverse Jacobian matrix which does not exist for kinematically redundant manipulators.

In applications where there are obstacles in the working area, extra joints may be required to carry out the task. By mounting a small and fast manipulator on a

Received 23 March 1987.

$\dagger$ This paper was presented at the IFAC Symposium on Robotic Control, Barcelona, Spain 6-8 November 1985. This paper is reprinted with the permission of IFAC.

$\ddagger$ Division of Engineering Cybernetics, The Norwegian Institute of Technology, N-7034 Trondheim-NTH, Norway. 
large and slow positioning arm, high bandwidth and a large working area may be obtained. This makes the use of kinematically redundant manipulators interesting.

In this paper a robot control system for Cartesian trajectory tracking is developed using optimal control theory. The problems introduced by the transformation of the trajectory to joint coordinates are avoided by an appropriate choice of the state vector. This optimal control system may also be used for kinematically redundant manipulators. For this type of manipulator, singularities are avoided by choosing a suitable performance index in the optimal control problem. A state space model is first developed. Then this model is modified by means of non-linear feedback into a model which is suitable for the well-known optimal tracking problem with a linear model and a quadratic performance index. Application to kinematically redundant manipulators is then discussed. Finally, the tracking system is simulated for a simple planar kinematically redundant manipulator.

\section{The state space model}

The equations of motion for a general manipulator can be found from NewtonEuler's equation (Luh, Walker and Paul 1980b, Symon 1971). We consider a manipulator with $n$ joints. We have:

$$
M(q) \ddot{q}+V \dot{q}+n(q, \dot{q})+g(q)=\tau
$$

where

$q \quad$ vector representing the actual displacements of the $n$ joints

$M(q) \quad$ inertia matrix

$V \quad$ viscous friction matrix

$\boldsymbol{n}(\boldsymbol{q}, \dot{\boldsymbol{q}}) \quad$ vector defining Coriolis and centrifugal terms

$\boldsymbol{g}(\boldsymbol{q}) \quad$ vector defining the gravity terms

$\tau \quad$ vector of input generalized forces

Actuator models are given in the form:

$$
J_{a} \ddot{q}+C_{a} \dot{q}+\tau=B_{a} \boldsymbol{u}_{a}
$$

where $J_{a}, C_{a}$ and $B_{a}$ are constant diagonal matrices, $\boldsymbol{u}_{a}$ is the actuator control vector, and

$$
\left|u_{a, i}\right| \leqslant u_{a, i}^{\max } .
$$

The position and orientation of the hand is given by $p=[x, y, z, \phi, \theta, \psi]^{T}$ where $x, y, z$ are the Cartesian coordinates of the hand, while $\phi, \theta$ and $\psi$ are the Euler angles.

The relationship between the velocity $\dot{\boldsymbol{p}}$, in Cartesian space and the velocity, $\dot{\boldsymbol{q}}$, in joint space is given by (Whitney, 1972)

$$
\dot{\boldsymbol{p}}=J(\boldsymbol{q}) \dot{\boldsymbol{q}}
$$

where $J(q)$ is the Jacobian matrix defined by $J_{i j}=\partial p_{i} / \partial q_{j}$. The three first lines of $J(q)$ are easily found, while the three last lines which are associated with the orientation part of $p$, are found in two steps. First the angular velocity of the hand is expressed by

$$
\omega=J_{\omega E}\left(p_{E}\right) \dot{p}_{E}
$$


where $p_{E}=[\phi, \theta, \psi]^{T}$. $\omega$ is also given by

$$
\omega=J_{\omega q}(q) \dot{q}
$$

The three last lines of $J(q)$ is then found from

$$
\dot{p}_{E}=J_{\omega E}^{-1}\left(p_{E}\right) J_{\omega q}(q) \dot{q}
$$

When the classical Euler angles are used, $J_{\omega E}$ will be singular when $\theta=0$. This problem can be solved by switching to the roll, pitch and yaw type of Euler angles in the neighbourhood of such a singularity.

For Cartesian trajectory tracking, we choose the state vector $x=\left[x_{1}^{T}, x_{2}^{T}\right]^{T}$ where $x_{1}=p$, which is the position of the hand in Cartesian coordinates, and $x_{2}=$ $\dot{q}$, which is the joint space velocity.

Combining (1), (2) and (3), we get the state space model

$$
E(q) \dot{x}=f\left(x, u_{a}\right)
$$

where

$$
E(q)=\left[\begin{array}{c:c}
I & 0 \\
\hdashline 0 & M(q)+J_{a}
\end{array}\right]
$$

$I$ is the identity matrix and

$$
f\left(x, u_{a}\right)=\left[\begin{array}{c}
J(q) x_{2} \\
-n\left(q, x_{2}\right)-\left(V+C_{a}\right) x_{2}-g(q)+B_{a} u_{a}
\end{array}\right]
$$

\section{Controller design}

If the reference is known in advance, optimal control theory may be applied off-line to compute the optimal control history. However, due to imperfect models and noise, there will be deviations from the optimal trajectory. These deviations must be compensated for by means of feedback control. In applications where the manipulator tracks a measured reference, the reference is not known in advance, and feedback control must be applied.

Optimal feedback control (Athans and Falb 1966, Bryson and Ho 1969) is only possible in certain cases. Here we will consider systems with a linear state space model and a quadratic performance index. The problem is then to formulate the manipulator tracking problem as a linear quadratic problem.

The state space model given by (4) is nonlinear and contains a large number of terms. We therefore choose the control vector

$$
u_{a}=B_{a}^{-1}\left[n\left(q, x_{2}\right)+\left(V+C_{a}\right) x_{2}+g(q)\right]+u
$$

This gives the simpler model

$$
\dot{x}=A(q) x+B(q) u
$$

where

$$
A(q)=\left[\begin{array}{c:c}
0 & J(q) \\
\hdashline 0 & 0
\end{array}\right]
$$

and

$$
B(q)=\left[\begin{array}{c}
0 \\
{\left[M(q)+J_{a}\right]^{-1} B_{a}}
\end{array}\right]
$$


The inertia matrix $M(q)+J_{a}$ is positive definite and therefore non-singular for all $q$. The model (6) is discretized by the Euler method. The discrete-time model is

$$
\boldsymbol{x}(k+1)=\Phi[\boldsymbol{q}(k)] \boldsymbol{x}(k)+\Gamma[q(k)] \boldsymbol{u}(k)
$$

We want to track a trajectory given by $r=\left[r_{1}^{T}, r_{2}^{T}\right]^{T}$ where $r_{1}$ is the Cartesian space position reference and $\boldsymbol{r}_{2}$ is the Cartesian space velocity reference, without excessive use of energy. The control vector $\boldsymbol{u}(k)$ is therefore chosen to minimize the performance index

$$
J=\lim _{N \rightarrow \infty} \frac{1}{N} \sum_{k=0}^{N-1} \frac{1}{2}\left[e^{T}(k) Q e(k)+u^{T}(k) P u(k)\right]
$$

where $Q$ and $P$ are diagonal matrices and $e(k)=y(k)-r(k) . y(k)$ is the measurement given by

$$
y(k)=D(q) x(k)
$$

where

$$
D(q)=\left[\begin{array}{c:c}
I & 0 \\
\hdashline 0 & J(q)
\end{array}\right]
$$

We see from (9) that $e(k)$ can be written as

$$
e(k)=\left[\begin{array}{c}
\Delta x_{1}(k) \\
x_{2}(k)-J(q) r_{2}(k)
\end{array}\right]
$$

where $\Delta x_{1}(k)$ is the position control deviation $\Delta x_{1}(k)=x_{1}(k)-r_{1}(k)$.

The optimal control is then given by

$$
u(k)=G(k)\left[\begin{array}{c}
\Delta x_{1}(k) \\
x_{2}(k)
\end{array}\right]-P^{-1} \Gamma^{T}\left[I+\Gamma G(k) \Phi^{-1}\right]^{T} h(k+1)
$$

where

$$
G(k)=\left[P+\Gamma^{T} R(k+1) \Gamma\right]^{-1} \Gamma^{T} R(k+1) \Phi
$$

and $R(k)$ is the solution of the discrete matrix Ricatti equation

$$
R(k)=\Phi^{T} R(k+1)[\Phi+\Gamma G(k)]+D^{T} Q D
$$

with boundary value $R(N)=0$. The last term in (11) gives an optimal feedforward from the velocity reference. $h(k)$ is found from

$$
h(k)=(\Phi+\Gamma G)^{T} h(k+1)-\left[\begin{array}{c}
0 \\
J^{T}(q) Q_{22} r_{2}(k)
\end{array}\right]
$$

with boundary value $\boldsymbol{h}(N)=\mathbf{0} . Q_{22}$ is part of $Q$ :

$$
Q=\left[\begin{array}{c:c}
Q_{11} & 0 \\
\hdashline 0 & Q_{22}^{-}
\end{array}\right]
$$

This is derived by assuming the costate to be $\lambda(k)=R(k) x(k)+\boldsymbol{h}(k)$ in analogy with the continuous case (Athans and Falb, 1966).

We see from (13) and (14) that to generate the optimal control vector given by (12), we have to know future values of $\Phi(q), \Gamma(q)$ and $\boldsymbol{r}_{2}$. In most cases, these future values will be unknown. A solution to this problem is to assume for the purpose of 


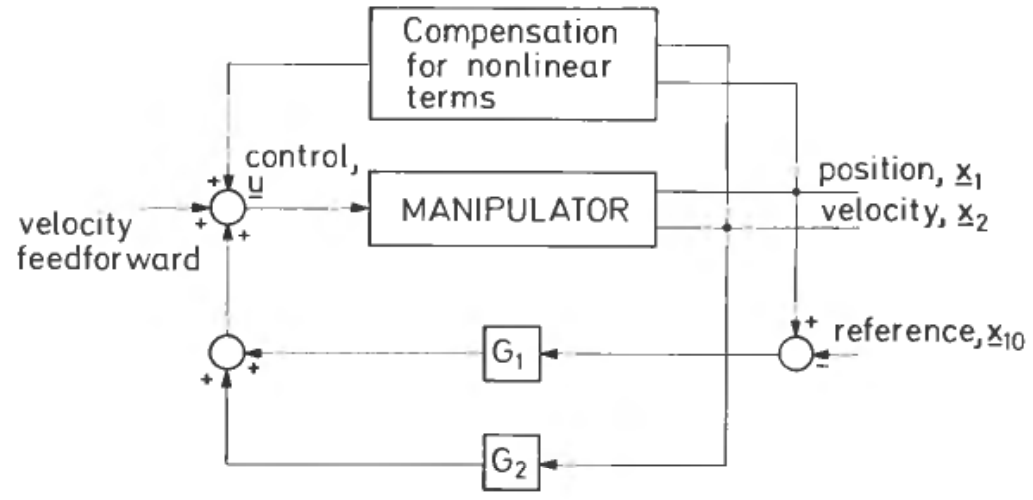

Figure 1. Control system.

computation that $\Phi, \Gamma$ and $r_{2}$ will remain constant. We then compute the stationary solution $R_{s}(k)$ of the discrete Ricatti equation (13) with $\Phi=\Phi[q(k)]$ and $\Gamma=\Gamma[q(k)]$. This is done by iterating (13) with $R_{s}(k-1)$ as a starting value. This iteration will converge in 1-3 iterations if $\Phi$ and $\Gamma$ are slowly changing. $G(k)$ is then given by (12) with $R(k+1)=R_{s}(k)$.

The stationary solution $h_{s}(k)$ of (14) is found by iterating (14) with $\Phi=\Phi[q(k)]$ and $\Gamma=\Gamma[q(k)]$ with $h_{s}(k-1)$ as a starting value. $\boldsymbol{u}(k)$ is then given by (11) with $\boldsymbol{h}(k+1)=h_{s}(k)$.

This results in a relatively simple control system which is shown in Fig. 1. The control system can also be used for kinematically redundant manipulators.

In the case of kinematically redundant manipulators, the number of joint variables is higher than the number of variables specifying the position of the hand. As a consequence of this, a state vector $x=\left[x_{1}^{T}, x_{2}^{T}\right]^{T}$ where $x_{1}$ is the position of hand, and $x_{2}$ is the joint space velocity, will not specify the joint coordinates of the manipulator.

We therefore augment $x_{1}$ to $x_{1 \mathrm{~A}}$ where $\operatorname{dim}\left(x_{1 \mathrm{~A}}\right)=n$, which is the number of joints. The new states in $x_{1 \mathrm{~A}}$ which will be denoted as $\tilde{\boldsymbol{x}}_{1}$, are positions that are chosen in such a way that $x_{1 \mathrm{~A}}$ specifies all joint coordinates. The state space model (6) is now augmented with

$$
\tilde{\tilde{x}}_{1}=\tilde{J}(q) x_{2}
$$

where

$$
\tilde{J}_{i j}=\partial \tilde{x}_{1, i} / \partial x_{2, j}
$$

We consider a redundant manipulator with a small and fast manipulator mounted on a large and slow arm. Small, high frequency movements should then be taken care of by the small manipulator, while large, low frequency movements should be attended to by the large arm. It is important that the small manipulator remains relatively close to the centre of its working area except for high frequency motion, even in the presence of a large control deviation.

This can be done by choosing $\tilde{\boldsymbol{x}}_{1}$ as the position of the hand relative to the base of the small manipulator. We then specify the reference $\tilde{r}_{1}$ to be the centre of the 


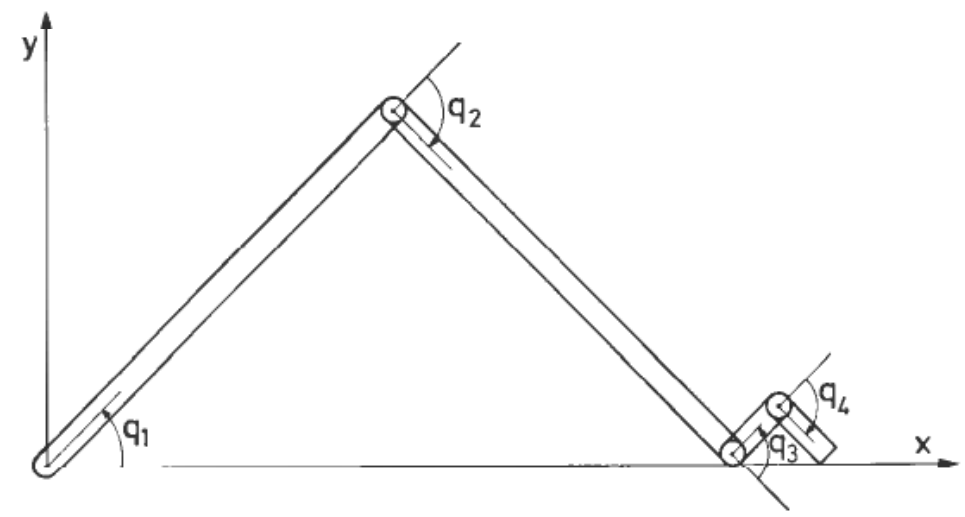

Figure 2. Four-link planar manipulator.

working area for the small manipulator. The performance index is then chosen as:

$$
J=\lim _{N \cdot \infty} \frac{1}{N} \sum_{k=0}^{N-1} \frac{1}{2}\left[e^{T}(k) Q e(k)+\tilde{e}^{T}(k) \tilde{Q} \tilde{e}(k)+u^{T}(k) P u(k)\right]
$$

where $\tilde{\boldsymbol{e}}=\tilde{\boldsymbol{x}}_{1}-\tilde{\boldsymbol{r}}_{1}$ and $\tilde{Q}$ is a diagonal matrix.

The optimal control for this case is found as described above, using $x_{1 \mathrm{~A}}$ instead of $x_{1}$.

\section{Application to kinematically redundant manipulator}

We consider the four-link planar manipulator in Fig. 2. The four joints have their axes of rotation along the $z$-axis. The joint angles are denoted $q_{1}, q_{2}, q_{3}$ and $q_{4}$.

The manipulator has four joints, and we want the position of the end of link four to track the reference. We therefore have a kinematically redundant system as the positioning of the end only requires two degrees of freedom.

We choose the state vector $x=\left[x_{1 \mathrm{~A}}^{T}, \boldsymbol{x}_{2}^{T}\right]^{T}$, where $\boldsymbol{x}_{1 \mathrm{~A}}=[x, y, \tilde{x}, \tilde{y}]^{T} . x$ and $y$ give the position of the end relative to the base, and $\tilde{x}$ and $\tilde{y}$ give the position of the end relative to the end of link two, which is the base of the small manipulator. $\boldsymbol{x}_{2}=\left[\omega_{1}, \omega_{2}, \omega_{3}, \omega_{4}\right]^{T}$ where $\omega_{1}, \omega_{2}, \omega_{3}$, and $\omega_{4}$ are the angular velocities of the four links.

The choice of $x_{2}=\omega$ gives a model with fewer terms than the model we get with $\overline{\boldsymbol{x}}_{2}=\left[\dot{q}_{1}, \dot{q}_{2}, \dot{q}_{3}, \dot{q}_{4}\right]^{T}$. The model is given by

$$
\dot{x}_{1 \mathrm{~A}}=\left[\begin{array}{rcrr}
-l_{1} S_{1} & -l_{2} S_{12} & -l_{3} S_{123} & -l_{4} S_{1234} \\
l_{1} C_{1} & l_{2} C_{12} & l_{3} C_{123} & l_{4} C_{1234} \\
0 & 0 & -l_{3} S_{123} & -l_{4} S_{1234} \\
0 & 0 & l_{3} C_{123} & l_{4} C_{1234}
\end{array}\right] x_{2}
$$




$$
\begin{aligned}
M(q) \dot{x}_{2}= & {\left[\begin{array}{c}
J_{12} S_{2} x_{6}^{2}+J_{13} S_{23} x_{7}^{2}+J_{14} S_{234} x_{8}^{2} \\
-J_{12} S_{2} x_{5}^{2}+J_{23} S_{3} x_{7}^{2}+J_{24} S_{34} x_{8}^{2} \\
-J_{13} S_{23} x_{5}^{2}-J_{23} S_{3} x_{6}^{2}+J_{34} S_{4} x_{8}^{2} \\
-J_{14} S_{234} x_{5}^{2}-J_{24} S_{34} x_{6}^{2}-J_{34} S_{4} x_{7}^{2}
\end{array}\right] } \\
& +\left[\begin{array}{rrrr}
1 & -1 & 0 & 0 \\
0 & 1 & -1 & 0 \\
0 & 0 & 1 & -1 \\
0 & 0 & 0 & 1
\end{array}\right]\left[\begin{array}{l}
u_{a 1} \\
u_{a 2} \\
u_{a 3} \\
u_{a 4}
\end{array}\right] .
\end{aligned}
$$

where

$$
M(q)=\left[\begin{array}{cccc}
J_{11} & J_{12} C_{2} & J_{13} C_{23} & J_{14} C_{234} \\
J_{12} C_{2} & J_{22} & J_{23} C_{3} & J_{24} C_{34} \\
J_{13} C_{23} & J_{23} C_{3} & J_{33} & J_{34} C_{4} \\
J_{14} C_{234} & J_{24} C_{34} & J_{34} C_{4} & J_{44}
\end{array}\right],
$$

$l_{1}, l_{2}, l_{3}$ and $l_{4}$ are the link lengths and $J_{i j}$ are the appropriate moments of inertia. Here $C_{i, \ldots, j}=\cos \left(q_{i}+\cdots+q_{j}\right)$ and $S_{i, \ldots, j}=\left(q_{i}+\cdots+q_{j}\right)$.

The four links are homogeneous beams. Links one and two are $1 \mathrm{~m}$ long, and the mass of each of them is $20 \mathrm{~kg}$. Links three and four are $0.1 \mathrm{~m}$, and the mass of each of them is $1 \mathrm{~kg}$.

The weight matrices were chosen as

$$
Q=\operatorname{diag}\left(1 \times 10^{8}, 1 \times 10^{8}, 1 \times 10^{4}, 1 \times 10^{4}, 1 \times 10^{4}, 1 \times 10^{4}\right)
$$

and

$$
P=\operatorname{diag}(0 \cdot 01,0 \cdot 01,1,1)
$$

With these weight matrices, a control deviation of $0.1 \mathrm{~mm}$ contributes as much to the performance index as a velocity deviation of $10 \mathrm{~mm} / \mathrm{s}$, a position deviation for the small manipulator of $10 \mathrm{~mm}$, a torque of $10 \mathrm{Nm}$ on the inner joints, or a torque of $1 \mathrm{Nm}$ on the outer joints.

The position reference $\tilde{\boldsymbol{r}}_{1}$ of the small manipulator was set to $\tilde{\boldsymbol{r}}_{1}=[0 \cdot 1414,0]^{T}$.

In a simulation experiment, the end of link four tracked a reference given by

$$
r_{1}=\left[\begin{array}{c}
1 \cdot 883+0.01 \sin [(2 \pi / 0 \cdot 2) t-\pi / 2] \\
0.1 t
\end{array}\right]
$$

which is shown in Fig. 3. This reference has a high frequency motion in the $x$ direction and a low frequency motion in the $y$ direction. The initial state was

$$
\boldsymbol{x}(0)=[1 \cdot 873,0,0 \cdot 1414,0,0,0,0,0]^{T}
$$

which corresponds to $q_{1}=30^{\circ}, q_{2}=-60^{\circ}, q_{3}=75^{\circ}$, and $q_{4}=-90^{\circ}$.

In Fig. 4 , we see the reference and the position in the $x$ direction. The control deviation is less than $0.2 \mathrm{~mm}$.

In Fig. 5 we see the position in the $y$ direction together with the contribution to the $y$ direction from the two inner and outer joints. We see that the outer joints take care of the initial movement, while the inner joints attend to the low frequency movement.

In Fig. 6 the contribution to the $x$ direction is shown for the two inner and outer joints. We see that the amplitude of the $x$ position of the end of link two is $3 \mathrm{~mm}$, 


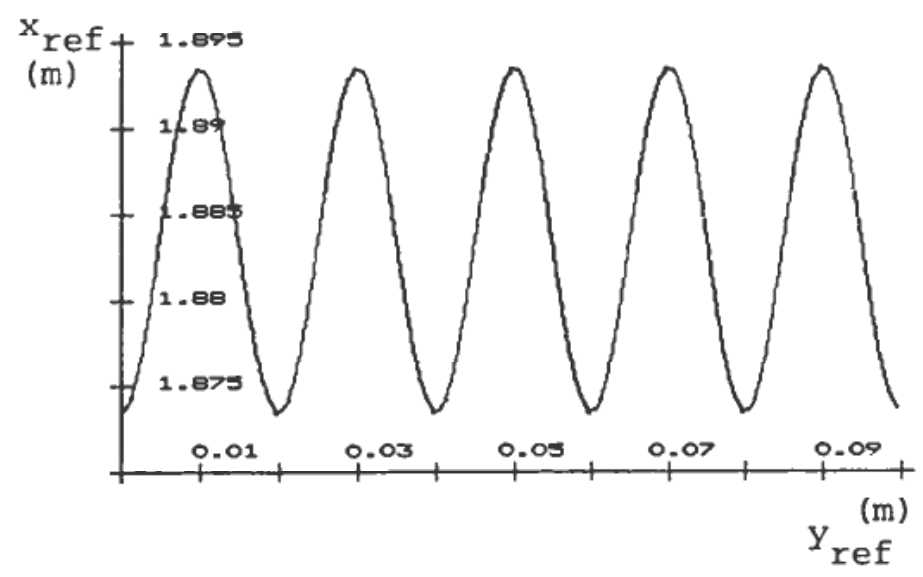

Figure 3. Reference.

while the small manipulator has an amplitude of $20 \mathrm{~mm}$ in the $x$ direction. The joint angles are shown in Fig. 7. The control variables are well-behaved. Here, we have considered joint torques as our controls. We clearly see that the small manipulator takes care of high frequency motion, while the two inner joints attend to slow and large movements.

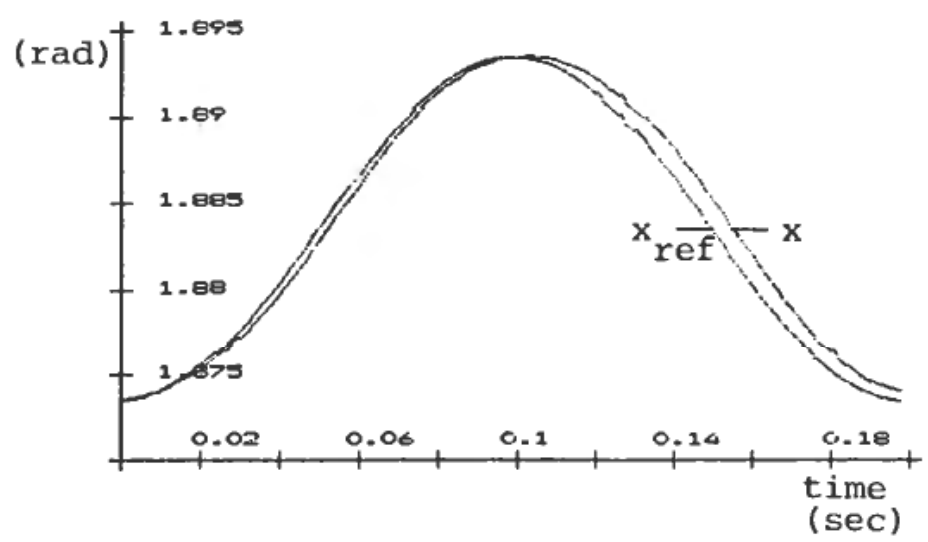

Figure 4. Reference and position in $x$ direction (expanded time scale).

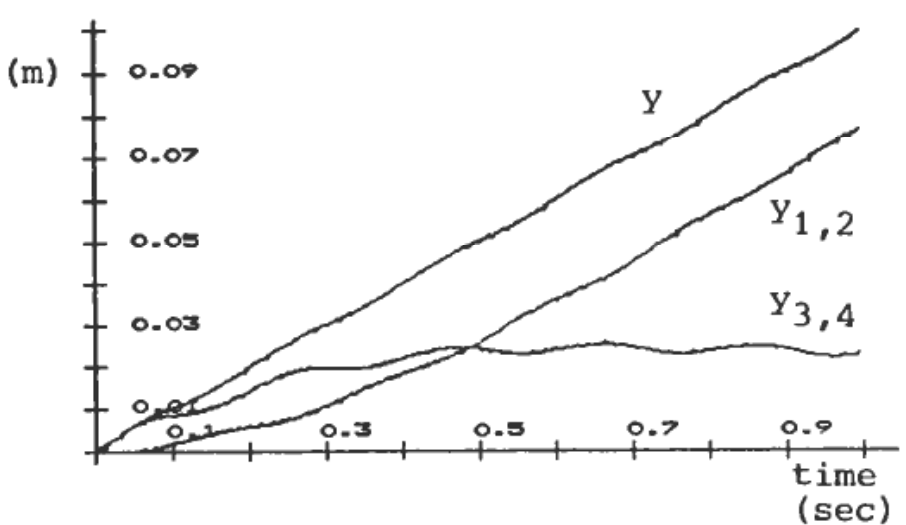

Figure 5. Position in $y$ direction, position $y_{1,2}$ of the end of link two, and position $y_{3,4}$ of the end of link four relative to the end of link two. 

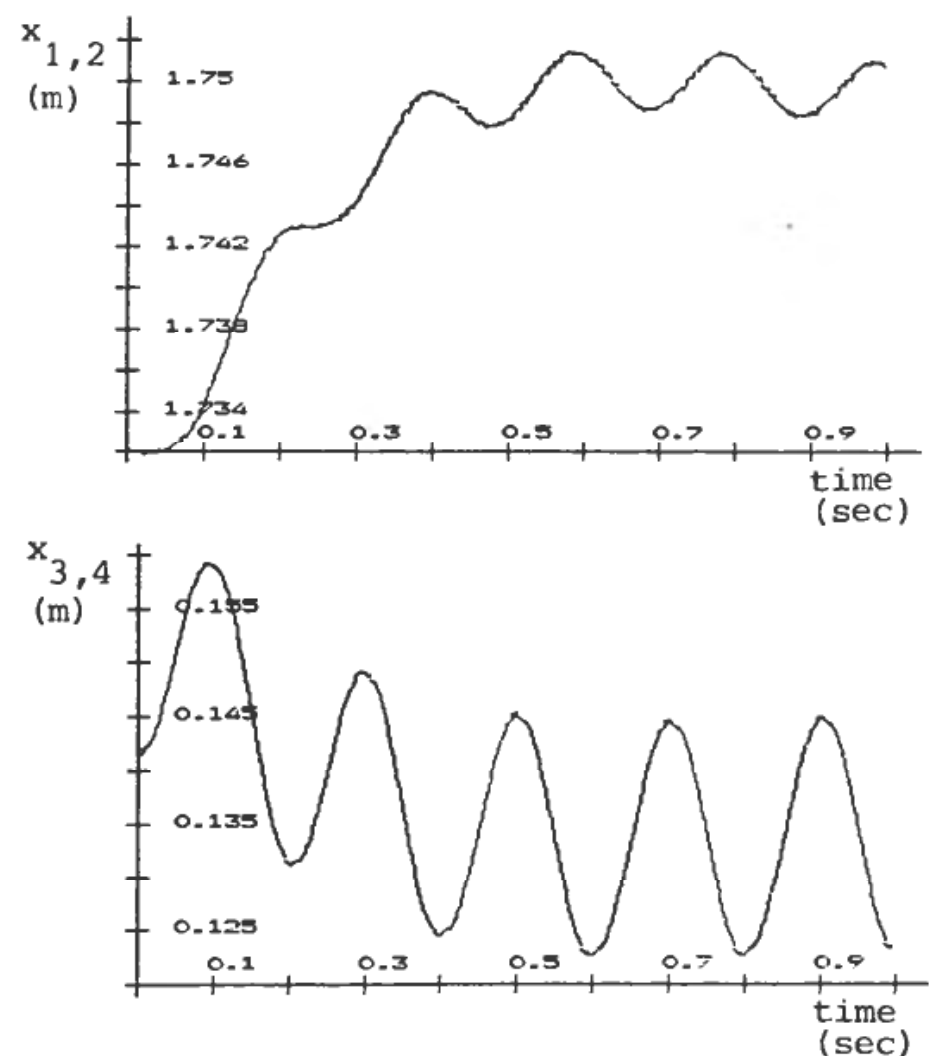

Figure 6. Position $x_{1,2}$ of the end of link two and position $x_{3,4}$ of the end of link four relative to the end of link two.

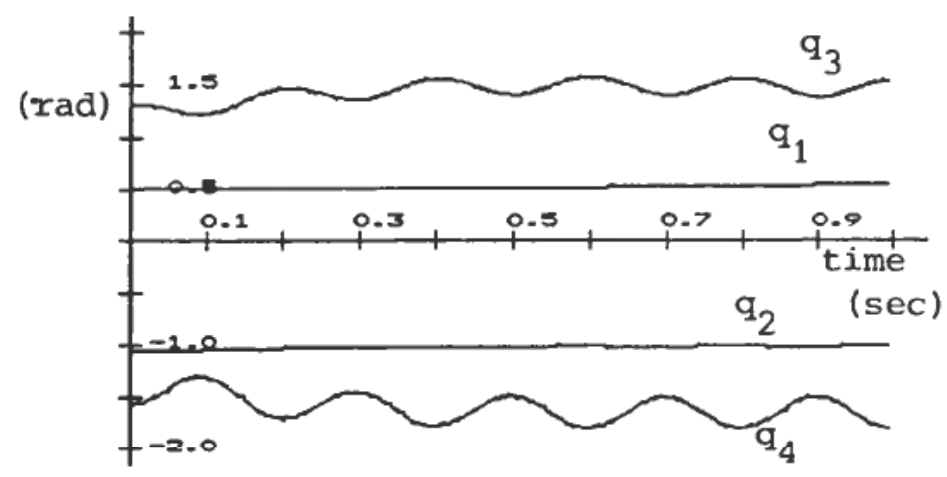

Figure 7. Joint angles. 

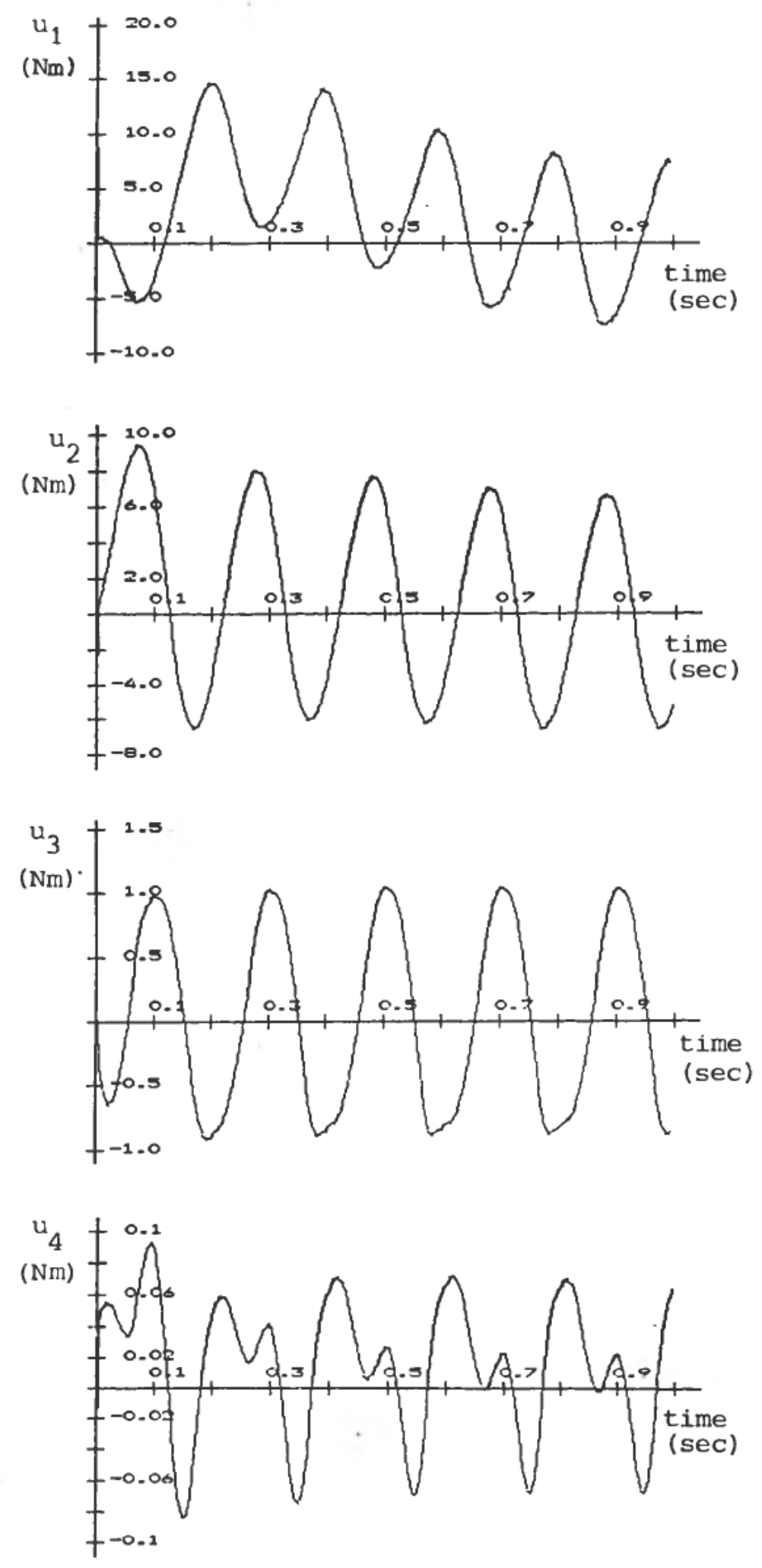

Figure 8. Control vector. 


\section{Conclusion}

A Cartesian trajectory tracking system for manipulators has been developed using optimal control theory. The tracking system can be applied to kinematically redundant manipulators. Simulation of the system was carried out using a four-link planar manipulator. A sinusoidal reference with an amplitude of $2 \mathrm{~mm}$ was tracked at a distance of $1.8 \mathrm{~m}$ from the base. The control deviation was less than $0.2 \mathrm{~mm}$ with moderate torques from the motors.

\section{REFERENCES}

Athans, M. and Falb, P. (1966). Optimal Control (McGraw-Hill, New York). Bryson, A. E. and Ho, Y. C. (1969). Applied Optimal Control (Blaisdell, New York).

LUH, J. Y. S. (1983). Conventional controller design for industrial robots-a tutorial. I.E.E.E. Trans. Syst., Man \& Cybernetics, 13, 298-316.

LuH, J. Y. S., Walker, M. W., and Paul, R. P. C. (1980a). Resolved acceleration control of mechanical manipulators. I.E.E.E. Trans. Autom. Control, 25, 468-474.

LuH, J. Y. S., Walker, M. W., and Paul, R. P. C. (1980b). On-line computational scheme for mechanical manipulators. J. Dynamic Systems, Measurement and Control, 102, 69-76.

PAUL, R. P. C. (1979). Manipulator cartesian path control. I.E.E.E. Trans. Syst., Man \& Cybernetics, 9, 702-711.

SARIDIS, G. N. (1983). Intelligent robotic control. I.E.E.E. Trans. Autom. Control, 28, 547-557.

Symon, K. R. (1971). Mechanics. (Addison Wesley, Reading, Massachusetts).

TARN, T. J., BEJCZY, A. K., IsIDORI, A., and CHEN, Y. (1984). Nonlinear feedback in robot arm control. Proc. 23rd I.E.E.E. Conference on Decision \& Control, Las Vegas, Nevada, Dec. 12-14, 1984.

TAYLOR, R. H. (1979). Planning and execution of straight line manipulator trajectories. IBM J. Res. \& Develop., 23, 253-264.

WhITNEY, D. E. (1972). The mathematics of coordinated control of prosthetic arms and manipulators. J. Dynamic Systems, Measurement and Control, 94, 303-309. 